This item was submitted to Loughborough's Research Repository by the author.

Items in Figshare are protected by copyright, with all rights reserved, unless otherwise indicated.

\title{
Theory of the charged Bose gas: Bose-Einstein condensation in an ultrahigh
}

\section{magnetic field}

PLEASE CITE THE PUBLISHED VERSION

PUBLISHER

(C) American Physical Society

LICENCE

CC BY-NC-ND 4.0

REPOSITORY RECORD

Alexandrov, A.S., W.H. Beere, and V.V. Kabanov. 2019. "Theory of the Charged Bose Gas: Bose-einstein Condensation in an Ultrahigh Magnetic Field". figshare. https://hdl.handle.net/2134/1315. 


\title{
Theory of the charged Bose gas: Bose-Einstein condensation in an ultrahigh magnetic field
}

\author{
A. S. Alexandrov \\ Interdisciplinary Research Center in Superconductivity, University of Cambridge, Madingley Road, Cambridge CB3 OHE, \\ United Kingdom \\ and Department of Physics, Loughborough University, Loughborough LE11 3TU, United Kingdom \\ W. H. Beere \\ Interdisciplinary Research Center in Superconductivity, University of Cambridge, Madingley Road, Cambridge CB3 OHE, \\ United Kingdom \\ V. V. Kabanov \\ Interdisciplinary Research Center in Superconductivity, University of Cambridge, Madingley Road, Cambridge CB3 OHE, \\ United Kingdom \\ and Frank Laboratory of Neutron Physics, Joint Institute for Nuclear Research, Dubna, Russia
}

(Received 25 March 1996; revised manuscript received 8 July 1996)

\begin{abstract}
The Bogoliubov-de Gennes equations and the Ginzburg-Landau-Abrikosov-Gor'kov-type theory are formulated for the charged Bose gas (CBG). The theory of the Bose-Einstein condensation of the CBG in a magnetic field is extended to ultralow temperatures and ultrahigh magnetic fields. A low-temperature dependence of the upper critical field $H_{c 2}(T)$ is obtained both for the particle-impurity and particle-particle scattering. The normal-state collective plasmon mode in ultrahigh magnetic fields is studied. [S0163-1829(96)08845-5]
\end{abstract}

\section{INTRODUCTION}

A charged Coulomb Bose gas (CBG) recently became of particular interest motivated by the bipolaron theory of hightemperature superconductivity. ${ }^{1}$ A long time ago Schafroth ${ }^{2}$ demonstrated that an ideal gas of charged bosons exhibits the Meissner-Ochsenfeld effect below the ideal Bose-gas condensation temperature. Later on, the one-particle excitation spectrum at $T=0$ was calculated by Foldy, ${ }^{3}$ who worked at zero temperature using the Bogoliubov ${ }^{4}$ approach. The Bogoliubov method leads to the result that the ground state of the system has a negative correlation energy, whose magnitude increases with the density of bosons. Perhaps more interesting is the fact that the elementary excitations of the system have, for small momenta, energies characteristic of plasma oscillations which pass over smoothly for large momenta to the energies characteristic of single-particle excitations. Further investigations have been carried out at or near $T_{c}$, the transition temperature for the gas. These works have been concerned with the critical exponents ${ }^{5}$ and the change in the transition temperature from that of the ideal gas. ${ }^{5,6}$ The random phase approximation (RPA) dielectric response function and screening in the CBG have been studied in the high-density limit, ${ }^{7}$ including a low-dimensional [twodimensional (2D)] CBG ${ }^{8,9}$ The theory of the CBG beyond the lowest-order Bogoliubov approximation was discussed by Lee and Feenberg ${ }^{10}$ and by Brueckner. ${ }^{11}$ They obtained the next-order correction to the ground-state energy. Woo and $\mathrm{Ma}^{12}$ calculated numerically the correction to the Bogoliubov excitation spectrum. Alexandrov ${ }^{13}$ found the critical magnetic field $H_{c 2}(T)$, at which the CBG is condensed. The predicted temperature dependence of $H_{c 2}(T)$ was observed both in low- $T_{c}$ and high- $T_{c}$ cuprates,${ }^{14}$ where the coherence volume estimated from the heat capacity measurements is comparable with or even less than the unit cell volume. This favors a charged $2 e$ Bose liquid as a plausible microscopic scenario for the ground state. ${ }^{1}$

Our objective is the theory of the CBG in a magnetic field. In this paper we first extend to finite temperatures the BdG-type equations derived earlier ${ }^{15}$ for $T=0$. Then we analyze the linearized Ginzburg-Landau-type equation for the order parameter and formulate the condition of the BoseEinstein condensation in a homogeneous magnetic field. By the use of the sum rule we calculate the upper critical field $H_{c 2}(T)$ both for a short-range particle-impurity and longrange particle-particle Coulomb interactions at low temperatures. The plasmon dispersion of CBG in the ultrahigh magnetic field is analyzed as well.

\section{BOGOLIUBOV-de GENNES EQUATIONS FOR THE CBG AT FINITE TEMPERATURES}

The superfluid properties of charged bosons as well as their excitation spectrum and the response function can be studied by the use of the Bogoliubov-de Gennes- (BdG-) type equations, fully taking into account the interaction of quasiparticles with the condensate. ${ }^{15}$ The Hamiltonian of charged bosons on an oppositely charged background (to ensure charge neutrality) in an external field with the vector potential $\mathbf{A}(\mathbf{r}, t)$ is given by

$$
\begin{aligned}
H= & \int d \mathbf{r} \psi^{\dagger}(\mathbf{r})\left[-\frac{\left(\nabla-i e^{*} \mathbf{A}\right)^{2}}{2 m}-\mu\right] \psi(\mathbf{r}) \\
& +\frac{1}{2} \int d \mathbf{r} \int d \mathbf{r}^{\prime} V\left(\mathbf{r}-\mathbf{r}^{\prime}\right) \psi^{\dagger}(\mathbf{r}) \psi(\mathbf{r}) \psi^{\dagger}\left(\mathbf{r}^{\prime}\right) \psi\left(\mathbf{r}^{\prime}\right) .
\end{aligned}
$$

For 3D charged bosons of mass $m$ the Fourier component of the interaction potential $V(\mathbf{r})$ is $V(\mathbf{k})=4 \pi e^{* 2} / k^{2}$ with bosonic charge $e^{*}$. For a $2 \mathrm{D}$ system with a three- 
dimensional interaction $V(\mathbf{k})=2 \pi e^{* 2} / k$. Respecting electroneutrality one takes $V(\mathbf{k} \equiv 0)=0$, which is a consequence of the compensation of the boson-boson repulsion by the attraction due to a spatially homogeneous charged background. In this paper we will treat the purely academic problem of the charged Bose gas; however, we state that the results obtained are also applicable to the case of preformed electron pairs, which we postulate to exist in the high- $T_{c}$ cuprates. In the more realistic case of preformed pairs their hard core nature needs to be considered. This introduces an additional term missing from the equations given in this paper as we no longer have $V(\mathbf{k} \equiv 0)=0$ and instead have $V(\mathbf{k} \equiv 0)=$ const. When considering the self-energy, as we shall do later in the paper, it can be seen that this zeromomenta interaction term will give a constant contribution to the self-energy, which amounts to a renormalization of the chemical potential. The hard core nature of the pairs also gives a constant contribution to the Fourier component of the interaction potential at all momenta. The most significant part of the interaction is Coulomb interaction as can be seen from Eq. (15), where, if we include the hard core interaction as well as the Coulomb interaction, the main result-that the excitation spectrum contains a plasma gap-is unchanged. The consequences of including only the hard core interaction have been discussed earlier by Alexandrov et al. ${ }^{16}$ One final point worth mentioning on the choice of the interaction potential deals with the screening within the system. Simply taking a screened Coulomb potential as our starting potential can lead to an erroneous double-counting result as discussed by Alexandrov and Beere. ${ }^{15}$ In this paper we start with the bare interaction potential and derive a self-consistent form of the self-energies, carefully taking into account the selfscreening of the interaction by the bosons in the high-density limit, $r_{s} \ll 1$ (see below). If we have in mind a metal with preformed pairs, to avoid their overlap, the density is also restricted in the upper limit. Here and further $\hbar=c=1$.

The equation of motion for the field operator $\psi$ is derived using this Hamiltonian,

$$
\begin{aligned}
i \frac{\partial}{\partial t} \psi(\mathbf{r}, t)= & {[H, \psi(\mathbf{r}, t)]=\left[-\frac{\left(\nabla-i e^{*} \mathbf{A}\right)^{2}}{2 m}-\mu\right] \psi(\mathbf{r}, t) } \\
& +\int d \mathbf{r}^{\prime} V\left(\mathbf{r}-\mathbf{r}^{\prime}\right) \psi^{\dagger}\left(\mathbf{r}^{\prime}, t\right) \psi\left(\mathbf{r}^{\prime}, t\right) \psi(\mathbf{r}, t) .
\end{aligned}
$$

If the interaction is weak, one can expect that the occupation numbers of one-particle states are not very much different from those in the ideal Bose gas. In particular the state with zero momentum $k=0$ remains macroscopically occupied and the corresponding Fourier component of the field operator $\psi(\mathbf{r})$ has an anomalously large matrix element between the ground states of the system containing $N+1$ and $N$ bosons. It is convenient to consider a grand canonical ensemble, introducing a chemical potential $\mu$. In this case the quantum state is a superposition of states $|N\rangle$ with slightly different total numbers of bosons. The weight of each state is a smooth function of $N$ which is practically constant near the average number $\bar{N}$ on the scale $\pm \sqrt{\bar{N}}$. Because $\psi$ changes the number of particles only by 1 its diagonal matrix element coincides with the off diagonal, calculated for the states with fixed $N=\bar{N}+1$ and $N=\bar{N}$. Following Bogoliubov ${ }^{4}$ one can separate the large diagonal matrix element $\psi_{0}$ from $\psi$ by treating the rest $\widetilde{\psi}$ as a small fluctuation:

$$
\psi(\mathbf{r}, t)=\psi_{0}(\mathbf{r}, t)+\widetilde{\psi}(\mathbf{r}, t) .
$$

The anomalous average $\psi_{0}(\mathbf{r}, t)=\langle\psi(\mathbf{r}, t)\rangle$ is equal to $\sqrt{n_{0}}$ in a homogeneous system, where $n_{0}$ is the condensate density.

Substituting the Bogoliubov displacement transformation, Eq. (3), into the equation of motion and collecting $c$-number terms of $\psi_{0}$ and supracondensate boson operators $\widetilde{\psi}$, we obtain a set of the BdG-type equations. The macroscopic condensate wave function, which plays the role of the order parameter, obeys the equation

$$
\begin{aligned}
i \frac{\partial}{\partial t} \psi_{0}(\mathbf{r}, t)=[ & \left.-\frac{\left(\nabla-i e^{*} \mathbf{A}\right)^{2}}{2 m}-\mu\right] \psi_{0}(\mathbf{r}, t)+\int d \mathbf{r}^{\prime} V\left(\mathbf{r}-\mathbf{r}^{\prime}\right) n\left(\mathbf{r}^{\prime}, t\right) \psi_{0}(\mathbf{r}, t)+\int d \mathbf{r}^{\prime} V\left(\mathbf{r}-\mathbf{r}^{\prime}\right)\left[\left\langle\widetilde{\psi}^{\dagger}\left(\mathbf{r}^{\prime}, t\right) \widetilde{\psi}(\mathbf{r}, t)\right\rangle \psi_{0}\left(\mathbf{r}^{\prime}, t\right)\right. \\
& \left.+\left\langle\widetilde{\psi}\left(\mathbf{r}^{\prime}, t\right) \widetilde{\psi}(\mathbf{r}, t)\right\rangle \psi_{0}^{*}\left(\mathbf{r}^{\prime}, t\right)\right] .
\end{aligned}
$$

Taking explicitly into account the interaction of supracondensate bosons with the condensate and applying the Hartree-Fock approximation for the interaction between supracondensate particles one obtains

$$
\begin{aligned}
& i \frac{\partial}{\partial t} \widetilde{\psi}(\mathbf{r}, t)=\left[-\frac{\left(\nabla-i e^{*} \mathbf{A}\right)^{2}}{2 m}-\mu\right] \widetilde{\psi}(\mathbf{r}, t)+\int d \mathbf{r}^{\prime} V\left(\mathbf{r}-\mathbf{r}^{\prime}\right) n\left(\mathbf{r}^{\prime}, t\right) \widetilde{\psi}(\mathbf{r}, t)+\int d \mathbf{r}^{\prime} V\left(\mathbf{r}-\mathbf{r}^{\prime}\right)\left[\psi_{0}^{*}\left(\mathbf{r}^{\prime}, t^{\prime}\right) \psi_{0}(\mathbf{r}, t)\right. \\
& \left.+\left\langle\widetilde{\psi^{\dagger}}\left(\mathbf{r}^{\prime}, t\right) \widetilde{\psi}(\mathbf{r}, t)\right\rangle\right] \widetilde{\psi}\left(\mathbf{r}^{\prime}, t\right)+\int d \mathbf{r}^{\prime} V\left(\mathbf{r}-\mathbf{r}^{\prime}\right)\left[\psi_{0}\left(\mathbf{r}^{\prime}, t\right) \psi_{0}(\mathbf{r}, t)+\left\langle\widetilde{\psi}\left(\mathbf{r}^{\prime}, t\right) \widetilde{\psi}(\mathbf{r}, t)\right\rangle\right] \widetilde{\psi}^{\dagger}\left(\mathbf{r}^{\prime}, t\right)+\int d \mathbf{r}^{\prime} V\left(\mathbf{r}-\mathbf{r}^{\prime}\right) \\
& \times\left[\widetilde{\psi^{\dagger}}\left(\mathbf{r}^{\prime}, t\right) \widetilde{\psi}\left(\mathbf{r}^{\prime}, t\right)-\left\langle\widetilde{\psi^{\dagger}}\left(\mathbf{r}^{\prime}, t\right) \widetilde{\psi}\left(\mathbf{r}^{\prime}, t\right)\right\rangle\right] \psi_{0}(\mathbf{r}, t)+\int d \mathbf{r}^{\prime} V\left(\mathbf{r}-\mathbf{r}^{\prime}\right)\left[\widetilde{\psi}^{\dagger}\left(\mathbf{r}^{\prime}, t\right) \widetilde{\psi}(\mathbf{r}, t)-\left\langle\widetilde{\psi}^{\dagger}\left(\mathbf{r}^{\prime}, t\right) \widetilde{\psi}(\mathbf{r}, t)\right\rangle\right] \psi_{0}\left(\mathbf{r}^{\prime}, t\right) \\
& +\int d \mathbf{r}^{\prime} V\left(\mathbf{r}-\mathbf{r}^{\prime}\right)\left[\widetilde{\psi}\left(\mathbf{r}^{\prime}, t\right) \widetilde{\psi}(\mathbf{r}, t)-\left\langle\widetilde{\psi}\left(\mathbf{r}^{\prime}, t\right) \widetilde{\psi}(\mathbf{r}, t)\right\rangle\right] \psi_{0}^{*}\left(\mathbf{r}^{\prime}, t\right) .
\end{aligned}
$$


Here

$$
n(\mathbf{r}, t)=\left|\psi_{0}(\mathbf{r}, t)\right|^{2}+\left\langle\widetilde{\psi}^{\dagger}(\mathbf{r}, t) \tilde{\psi}(\mathbf{r}, t)\right\rangle
$$

is the boson density, and so the general sum rule

$$
\int d \mathbf{r} n(\mathbf{r}, t)=n_{B}
$$

is satisfied. Here $n_{B}$ is the number of bosons in the normalized volume, which is taken to be unity.

In the high-density limit $r_{s}=m e^{* 2} /\left(4 \pi n_{B} / 3\right)^{1 / 3} \ll 1$ and for the temperature close to zero the number of bosons pushed up from the condensate by the repulsion is small. Therefore the contribution of terms nonlinear in $\widetilde{\psi}$ is negligible. Applying a linear Bogoliubov transformation for $\widetilde{\psi}$,

$$
\widetilde{\psi}(\mathbf{r}, t)=\sum_{n} u_{n}(\mathbf{r}, t) \alpha_{n}+v_{n}^{*}(\mathbf{r}, t) \alpha_{n}^{\dagger}
$$

where $\alpha_{n}$ and $\alpha_{n}^{\dagger}$ are bosonic quasiparticle operators for the one-particle quantum state $n$, and omitting nonlinear terms, we obtain two coupled Schrödinger equations for the wave functions $u(\mathbf{r}, t)$ and $v(\mathbf{r}, t):^{.15}$

$$
\begin{aligned}
i \frac{\partial}{\partial t} u(\mathbf{r}, t)= & \left.-\frac{\left(\nabla-i e^{*} \mathbf{A}\right)^{2}}{2 m}-\mu\right] u(\mathbf{r}, t) \\
& +\int d \mathbf{r}^{\prime} V\left(\mathbf{r}-\mathbf{r}^{\prime}\right)\left[\left|\psi_{0}\left(\mathbf{r}^{\prime}, t\right)\right|^{2} u(\mathbf{r}, t)\right. \\
& \left.+\psi_{0}^{*}\left(\mathbf{r}^{\prime}, t\right) \psi_{0}(\mathbf{r}, t) u\left(\mathbf{r}^{\prime}, t\right)\right] \\
& +\int d \mathbf{r}^{\prime} V\left(\mathbf{r}-\mathbf{r}^{\prime}\right) \psi_{0}\left(\mathbf{r}^{\prime}, t\right) \psi_{0}(\mathbf{r}, t) v\left(\mathbf{r}^{\prime}, t\right)
\end{aligned}
$$

and

$$
\begin{aligned}
-i \frac{\partial}{\partial t} v(\mathbf{r}, t)= & \left.-\frac{\left(\nabla+i e^{*} \mathbf{A}\right)^{2}}{2 m}-\mu\right] v(\mathbf{r}, t) \\
& +\int d \mathbf{r}^{\prime} V\left(\mathbf{r}-\mathbf{r}^{\prime}\right)\left[\left|\psi_{0}\left(\mathbf{r}^{\prime}, t\right)\right|^{2} v(\mathbf{r}, t)\right. \\
& \left.+\psi_{0}\left(\mathbf{r}^{\prime}, t\right) \psi_{0}^{*}(\mathbf{r}, t)\right] v\left(\mathbf{r}^{\prime}, t\right) \\
& +\int d \mathbf{r}^{\prime} V\left(\mathbf{r}-\mathbf{r}^{\prime}\right) \psi_{0}^{*}\left(\mathbf{r}^{\prime}, t\right) \psi_{0}^{*}(\mathbf{r}, t) u\left(\mathbf{r}^{\prime}, t\right)
\end{aligned}
$$

There is also another sum rule

$\sum_{n}\left[u_{n}(\mathbf{r}, t) u_{n}^{*}\left(\mathbf{r}^{\prime}, t\right)-v_{n}(\mathbf{r}, t) v_{n}^{*}\left(\mathbf{r}^{\prime}, t\right)\right]=\delta\left(\mathbf{r}-\mathbf{r}^{\prime}\right)$

which retains the Bose commutation relations for all operators.

Unfortunately, the last set of BdG equations (9)-(11) is applied only for low temperatures and small magnetic fields, when the depletion of the condensate is small. As an example for the homogeneous case and $\mathbf{A}=0$ the excitation wave functions are plane waves

$$
\begin{aligned}
& u_{\mathbf{k}}(\mathbf{r}, t)=u_{\mathbf{k}} e^{i \mathbf{k} \cdot \mathbf{r}-i \epsilon_{\mathbf{k}} t}, \\
& v_{\mathbf{k}}(\mathbf{r}, t)=v_{\mathbf{k}} e^{i \mathbf{k} \cdot \mathbf{r}-i \epsilon_{\mathbf{k}} t},
\end{aligned}
$$

and the condensate wave function is $(\mathbf{r}, t)$ independent, $\psi_{0}=\sqrt{n_{0}}$. Thus the solution to Eq. (4) is

$$
\mu=V(\mathbf{k} \equiv 0)=0
$$

in the leading order in $r_{s}$, when the last term in Eq. (4) can be neglected. The excitation spectrum is that obtained by Foldy, ${ }^{3}$

$$
\epsilon_{\mathbf{k}}=\sqrt{\frac{k^{4}}{4 m^{2}}+\frac{k^{2} V(\mathbf{k}) n_{0}}{m}}
$$

with a gap $\omega_{p 0}=\sqrt{4 \pi e^{2} n_{0} / m}$, which is the classical plasma frequency for a plasma of density $n_{0}$.

At finite temperatures and (or) in a strong magnetic field we are left with the extremely complicated integrodifferential nonlinear equations (4)-(7).

\section{UPPER CRITICAL FIELD OF CBG: GENERAL FORMULATION}

The situation, however, is not hopeless in the region of the second-order phase transition near the upper critical field $H_{c 2}(T)$. In this region one can apply the expansion in powers of the order parameter $\psi_{0}(\mathbf{r}, t)$ to obtain the equation similar to that of the Ginzburg-Landau-Abrikosov-Gor'kov (GLAG) theory. ${ }^{19,20}$ In particular, the linearized equation (4) takes the form

$$
\begin{aligned}
i \frac{\partial}{\partial t} \psi_{0}(\mathbf{r}, t)= & \left.-\frac{\left(\nabla-i e^{*} \mathbf{A}\right)^{2}}{2 m}-\mu\right] \psi_{0}(\mathbf{r}, t) \\
& +\int d \mathbf{r}^{\prime} V\left(\mathbf{r}-\mathbf{r}^{\prime}\right) n\left(\mathbf{r}^{\prime}, t\right) \psi_{0}(\mathbf{r}, t) \\
& +\int d \mathbf{r}^{\prime} V\left(\mathbf{r}-\mathbf{r}^{\prime}\right)\left\langle\widetilde{\psi^{\dagger}}\left(\mathbf{r}^{\prime}, t\right) \widetilde{\psi}(\mathbf{r}, t)\right\rangle \psi_{0}\left(\mathbf{r}^{\prime}, t\right) .
\end{aligned}
$$

The last two terms are the Hartree-Fock corrections to the normal-state single-boson energy, respectively. This can be reduced to the renormalization of the normal-state singleboson energy spectrum $(m)$ and of the chemical potential, as discussed in the last section. One of the solutions to Eq. (16) is a trivial $\psi_{0}=0$. If this solution is compatible with the sum rule, Eq. (7), we have the normal state. On the other hand, if $\psi_{0} \neq 0$, the linearized equation (16) can be satisfied with the appropriate choice of the chemical potential (in a stationary homogeneous magnetic field). Therefore, the linearized equation for the order parameter does not determine the upper critical field at all. The upper critical field is determined by the sum rule, Eq. (7), as the lowest field at which this rule cannot be satisfied with $\psi_{0}=0,{ }^{13}$ rather than by the linearized GL equation of the GLAG theory, where $\mu \sim T_{c}-T$ is fixed.

Introducing the one-particle normal-state Green's function 


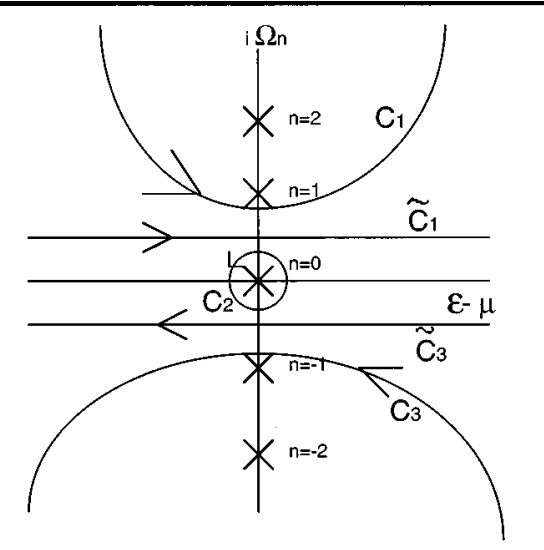

FIG. 1. Contour transformation of the sum rule.

$$
\mathcal{G}_{\nu}\left(i \Omega_{n}\right)=\frac{1}{i \Omega_{n}-\epsilon_{\nu}+\mu-\Sigma_{\nu}\left(i \Omega_{n}\right)},
$$

we can write the sum rule in the Matsubara representation as

$$
-T \sum_{n, \nu} \frac{e^{i \Omega_{n} 0^{+}}}{i \Omega_{n}-\epsilon_{\nu}+\mu-\Sigma_{\nu}\left(i \Omega_{n}\right)}=n_{B},
$$

where $\epsilon_{\nu}=\omega_{H}(N+1 / 2)+k_{z}^{2} / 2 m$ is the free-particle spectrum in a homogeneous magnetic field $H, \omega_{H}=e^{*} H / m, k_{z}$ is the $z$ component of the momentum, $N=0,1,2, \ldots, \Omega_{n}=2 \pi T n$, $n=0, \pm 1, \pm 2, \ldots$, and the self-energy $\Sigma_{\nu}\left(\Omega_{n}\right)$ takes into account the interaction terms. The complete set of the quantum numbers is $\nu=\left(N, k_{z}, k_{x}\right)$ with the energy degenerate for the quantum number $k_{x}$. It is convenient to replace the summation for the contour integral over $C_{1}, C_{2}$, and $C_{3}$ as shown in Fig. 1. Then shifting the contour to the real axis and by the analytical continuation of the Green's function to the upper $\left(G^{R}\right)$ and lower $\left(G^{A}\right)$ half-planes the sum rule is given by

$$
\int_{\mu+0}^{\infty} \frac{d \epsilon \rho(\epsilon, H)}{\exp [(\epsilon-\mu) / T]-1}=n_{B},
$$

where $G_{\nu}^{A, R}(\epsilon)=\left[\epsilon-\epsilon_{\nu}-\Sigma_{\nu}^{A, R}(\epsilon)\right]^{-1}$, and

$$
\rho(\epsilon, H)=\frac{1}{2 \pi i} \sum_{\nu}\left[G_{\nu}^{A}(\epsilon)-G_{\nu}^{R}(\epsilon)\right]
$$

is the one-particle density of states (DOS) in the magnetic field, with the edge of the spectrum, $E_{c}$, determined by the condition $\rho\left(E_{c}, H\right)=0$. The integral over $C_{2}$ is compensated for by the opposite sign contribution of $\widetilde{C}_{1}$ and $\widetilde{C}_{3}$ (Fig. 1). This and the condition $\mu \leqslant E_{c}$ determine the lower limit in Eq. (19) as $\mu+0$. It is apparent now that the upper critical field is determined by Eq. (19) with $H=H_{c 2}$ and $\mu=E_{c}$. Hence, the problem of the Bose-Einstein condensation in the magnetic field is reduced to the calculation of the normalstate DOS, Eq. (20). In particular, for free particles we have $\sum_{\nu}^{A, R}(\epsilon)= \pm i \delta, \delta \rightarrow+0$, so that

$\rho(\epsilon, H)=\frac{\sqrt{2} m^{3 / 2} \omega_{H}}{4 \pi^{2}} \operatorname{Re} \sum_{N=0}^{\infty}\left[\epsilon-\omega_{H}(N+1 / 2)\right]^{-1 / 2}$, and $E_{c}=\omega_{H} / 2$. Substituting this DOS into the sum rule we obtain the familiar Schafroth result ${ }^{2}$

$$
H_{c 2}(T)=\frac{4 \pi^{2} n_{B}}{e^{*} \sqrt{2 m} T \int_{0}^{\infty} d \epsilon \epsilon^{-3 / 2}} \equiv 0 .
$$

The condensation of ideal charged bosons in the magnetic field is impossible which is a consequence of the onedimensional motion in the lowest Landau level. To obtain a finite value of $\mathrm{H}_{c 2}$ one should go beyond the Hartree-Fock approximation, taking into account the broadening of the Landau levels. If this broadening is to have an effect on $H_{c 2}$, then it must act to make the density of states converge to zero at $E_{c}$, ensuring that the integral in Eq. (19) will be finite.

\section{CONDENSATION OF BOSONS SCATTERED BY IMPURITIES}

Here we extend the earlier study ${ }^{13}$ of the condensation of charged bosons scattered by impurities to low temperatures and ultrahigh magnetic fields, where the Born approximation for a single-impurity scattering fails.

In the lowest order in impurity concentration the selfenergy $\Sigma$ in a magnetic field is expressed in terms of a single-impurity $t$ matrix ${ }^{17}$

$$
\begin{gathered}
\Sigma_{\nu, \nu^{\prime}}(\epsilon)=\left[\hat{G}^{(0)}(\epsilon)\right]_{\nu, \nu^{\prime}}^{-1}-[\hat{G}(\epsilon)]_{\nu, \nu^{\prime}}^{-1}, \\
\Sigma_{\nu, \nu^{\prime}}(\epsilon)=n_{\mathrm{im}} t_{\nu, \nu^{\prime}}(\boldsymbol{\epsilon}),
\end{gathered}
$$

where $n_{\text {im }}$ is the concentration of impurities. This expression corresponds to the summation of all noncrossing diagrams (ladder approximation). ${ }^{18,19,21-23}$ The $t$ matrix in the magnetic field is derived by the use of the general formalism ${ }^{17}$ fully taking into account the multiple scattering:

$$
t_{\alpha, \beta}=\frac{2 \pi f}{m} \int d \mathbf{r} \frac{\phi_{\alpha}^{*}(\mathbf{r}) \phi_{\beta}(\mathbf{r})}{1+\frac{2 \pi f}{m} \sum_{\nu, \nu^{\prime}} \phi_{\nu}^{*}(\mathbf{r}) \phi_{\nu^{\prime}}(\mathbf{r}) G_{\nu, \nu^{\prime}}(\boldsymbol{\epsilon})},
$$

where $\phi_{\nu}(\mathbf{r})$ is the one-particle wave function in the magnetic field. In the Landau representation with the magnetic field along the $z$ axis $\phi_{\nu}(\mathbf{r})$ is given by

$$
\begin{aligned}
\phi_{\nu}(\mathbf{r})= & \frac{1}{\pi^{1 / 4} a_{H}^{1 / 2} \sqrt{2^{N} N !}} \exp \left[i\left(k_{x} x+k_{z} z\right)\right. \\
& \left.-\frac{1}{2}\left(\frac{y-y_{0}}{a_{H}}\right)^{2}\right] H_{N}\left(\frac{y-y_{0}}{a_{H}}\right) .
\end{aligned}
$$

$H_{N}(y)$ is a Hermite polynomial, $y_{0}=k_{x} a_{H}^{2}$ is the center of the cyclotron motion, and $a_{H}=1 / \sqrt{e^{*} H}$ is the magnetic length. $G_{\nu, \nu^{\prime}}(\epsilon)$ is the one-particle Green's function averaged over the position of the impurities, and $f$ is the scattering amplitude of a particle with zero energy in zero magnetic field. This equation is similar to that discussed by Skobov ${ }^{22}$ and Magarill and Savvinykh. ${ }^{23}$

It should be pointed out that the self-energy $\Sigma_{\nu, \nu^{\prime}}(\epsilon)$ has diagonal $\left(\nu=\nu^{\prime}\right)$ and nondiagonal $\left(\nu \neq \nu^{\prime}\right)$ components. Both diagonal and nondiagonal components contribute to the density of states, $N(\epsilon)=\operatorname{Tr} \operatorname{Im} G_{\nu, \nu}(\epsilon) / \pi$. 
There are two dimensionless parameters in this problem. The first is the ratio of the scattering amplitude $f$ to the magnetic length $a_{H}$. At low temperatures, where $H_{c 2}$ is large, this parameter is not small. Therefore, at low temperatures, one has to go beyond the Born approximation for a single-impurity scattering by the use of Eq. (25). The second parameter is the ratio of the mean-free path, $l=v \tau$, to the magnetic length. This is the same as $\omega_{H} \tau$ for particles at the lowest Landau level where $v \approx\left(m a_{H}\right)^{-1}$. Here $1 / \tau$ is the order of the collision broadening of the Landau levels, which depends on the magnetic field as $H^{2 / 3}$ (see below). As a result in the low-temperature limit, when $H_{c 2}$ is large, the nondiagonal components of the Green's function $G_{\nu, \nu^{\prime}}$ are small when compared with the diagonal components as $1 /\left(\omega_{H} \tau\right) \ll 1$. It should be pointed out that the $t$ matrix, Eq. (25), is essentially nondiagonal, but the contribution of the nondiagonal elements of the $t$ matrix to the density of states is small, as $1 /\left(\omega_{H} \tau\right)$.

The equation for the diagonal part of the self-energy, $\Sigma_{\alpha}(\epsilon)$, has the following form:

$$
\Sigma_{\alpha}(\boldsymbol{\epsilon})=\frac{2 \pi f n_{\mathrm{im}}}{m} \int d \mathbf{r} \frac{\phi_{\alpha}^{*}(\mathbf{r}) \phi_{\alpha}(\mathbf{r})}{1+(2 \pi f / m) \Sigma_{\nu} \phi_{\nu}^{*}(\mathbf{r}) \phi_{\nu}(\mathbf{r}) G_{\nu}(\boldsymbol{\epsilon})} .
$$

By the use of the fact that in a homogeneous system $G_{\nu}$ is $k_{x}$ independent one can perform the summation over $k_{x}$ in the denominator. Then integrating over $\mathbf{r}$ we obtain the following equation in the ultraquantum limit $(N=0)$ :

$$
\sigma(\widetilde{\boldsymbol{\epsilon}})=\gamma \frac{1}{1+i / \sqrt{\widetilde{\boldsymbol{\epsilon}}-\sigma(\widetilde{\boldsymbol{\epsilon}})}},
$$

where $\quad \gamma=2 \pi f n_{\mathrm{im}} / \epsilon_{0} m, \quad \sigma=\Sigma / \epsilon_{0}, \quad \tilde{\boldsymbol{\epsilon}}=\epsilon / \epsilon_{0}, \quad$ and $\epsilon_{0}=f^{2} / 2 m a_{H}^{4}$.

The density of states is given by

$$
N(\epsilon)=\frac{\sqrt{2} m^{3 / 2} \omega_{H}}{4 \pi^{2}} \operatorname{Re} \frac{1}{\sqrt{\epsilon-\Sigma(\epsilon)}} .
$$

Solving the cubic equation (28) we find the edge, where a nonzero density of states appears, as

$$
\epsilon_{c} \equiv \frac{E_{c}}{\epsilon_{0}}=A^{1 / 3}+\frac{1 / 9-3 \gamma}{A^{1 / 3}}+\gamma-2 / 3,
$$

with $A=1+(3 / 2)^{3}\left\{\sqrt{x\left[\gamma+(2 / 3)^{3}\right]^{3}}-(10 / 27-\gamma)^{2}\right\}$. Expanding the imaginary part of the self-energy near the edge we arrive with the following expression for $N(\epsilon)$ :

$$
N(\epsilon)=\frac{\sqrt{2} m^{3 / 2} \omega_{H}}{4 \pi^{2} \epsilon_{0}} \sqrt{\epsilon-E_{c}} \sqrt{\frac{R^{2}(R+1)}{\epsilon_{c}(3 R+1)-\gamma}},
$$

where $R=-\left(6 \epsilon_{c}-2-6 \gamma\right) /\left(8 \epsilon_{c}+\gamma\right)$.

One can distinguish two limiting cases. The first one corresponds to the collision broadening of the Landau levels ${ }^{21-24}$ when the multiple scattering by a single impurity is negligible. This is $\gamma \gg 1$, and so

$$
\epsilon_{c}=\gamma-3 /(\gamma / 2)^{2 / 3}
$$

and

$$
N(\epsilon) \simeq N_{\mathrm{col}}(\epsilon)=\frac{m^{3 / 2} \omega_{H}}{\sqrt{6} 2^{1 / 3} \Gamma_{0}} \sqrt{\epsilon-E_{c}}
$$

where the parameter $\Gamma_{0}=\left(4 \pi f^{2} n_{\text {im }} / a_{H}^{2}\right)^{2 / 3} / 2 m$ is the collision broadening of the Landau levels. In this limit temperature dependence of the upper critical field has been found by Alexandrov: ${ }^{13,14}$

$$
H_{c 2} \approx \frac{\Phi_{0}}{2 \pi \xi^{2}}\left(T_{c_{0}} / T\right)^{9 / 2},
$$

with $\Phi_{0}=\pi / e$ the flux quantum, $T_{c_{0}} \simeq 3.3 n_{B}^{2 / 3} / m$, and the "coherence", length $\xi=\pi \sqrt{\left(e^{*} / 2 e\right)} / 3^{3 / 4} f^{2} n_{\text {im }}$ is proportional to the mean-free path in zero magnetic field.

When the temperature decreases the low-energy excitations become more important. Then the multiple scattering gives the leading contribution. In that case $(\gamma<<1)$ broadening of the Landau levels is determined by the parameter $\epsilon_{0}$, and so

$$
\begin{gathered}
\boldsymbol{\epsilon}_{c}=(\gamma / 2)^{2}, \\
N(\boldsymbol{\epsilon}) \simeq N_{\text {scat }}(\boldsymbol{\epsilon})=\frac{m^{3 / 2}}{4 \pi^{2} \sqrt{2} a_{H}^{6} n_{\mathrm{im}}^{2}} \sqrt{\boldsymbol{\epsilon}-E_{c}} .
\end{gathered}
$$

It should be pointed out that the ratio $N_{\text {col }}(\epsilon) / N_{\text {scat }}(\epsilon)$ $\propto\left(\Gamma_{0} / \epsilon_{0}\right)^{2}$. The temperature dependence of the upper critical field is different in that case and is given by

$$
H_{c 2} \approx \frac{\Phi_{0}}{2 \pi \xi^{2}}\left(T_{c_{0}} / T\right)^{1 / 2}
$$

where $\xi=\sqrt{e^{* / 2 e}}\left(2 n_{\text {im }}\right)^{-1 / 3}$

With the temperature lowering the upper critical field diverges. Therefore in the ultralow-temperature limit we have $\epsilon_{0} \gg \Gamma_{0}$. As a result the $T^{-4.5}$ divergence in Eq. (34) transforms into $T^{-0.5}$ in Eq. (37) when $T$ goes to zero. In this limit we also expect that the localization of bosons, due to the high magnetic field, in the random potential might be important.

\section{NORMAL-STATE PLASMON IN THE ULTRAQUANTUM LIMIT}

As discussed above the condensation temperature in a magnetic field is zero without scattering because the density of states diverges at low energy. In a clean system the selfenergy arising from the Coulomb scattering is the main source of the level broadening, resulting in a vanishing density of states at low energy and thus in a finite condensation temperature $T_{c}$. We can expect that at low temperatures density fluctuations of the $\mathrm{CBG}$, i.e., plasmons, play the role of impurities.

Plasmons are defined as poles of the renormalized Coulomb potential. We calculate the renormalized Coulomb potential as a summation series of polarization loops (Fig. 2), given by

$$
\mathcal{D}\left(\mathbf{q}, i \Omega_{n}\right)=\frac{V(\mathbf{q})}{1-V(\mathbf{q}) \Pi\left(\mathbf{q}, i \Omega_{n}\right)},
$$

where 


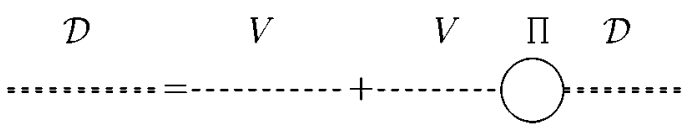

FIG. 2. Plasmon propagator.

$$
\Pi\left(\mathbf{q}, i \Omega_{n}\right)=T \sum_{i \Omega_{m}} \sum_{\nu, \nu^{\prime}}\left|I_{\nu}^{\nu^{\prime}}(\mathbf{q})\right|^{2} \mathcal{G}_{\nu}\left(i \Omega_{m}\right) \mathcal{G}_{\nu^{\prime}}\left(i \Omega_{m}+i \Omega_{n}\right)
$$

is the polarization loop in the Matsubara representation, and $I_{\nu}^{\nu^{\prime}}(\mathbf{q})$ is the matrix element defined as

$$
I_{\nu}^{\nu^{\prime}}(\mathbf{q})=\int d \mathbf{r} e^{i \mathbf{q} \cdot \mathbf{r}} \phi_{\nu}^{*}(\mathbf{r}) \phi_{\nu^{\prime}}(\mathbf{r}) .
$$

In the Landau representation, Eq. (26), one obtains

$$
\begin{aligned}
I_{\nu}^{\nu^{\prime}}(\mathbf{q})= & \delta_{k_{x}+q_{x}, k_{x}^{\prime} \delta_{k_{z}+q_{z}, k_{z}^{\prime}}\left(\frac{N^{\prime} !}{N !}\right)^{1 / 2}} \\
& \times\left[\frac{\left(-q_{x}+i q_{y}\right) a_{H}}{\sqrt{2}}\right]^{N-N^{\prime}} L_{N^{\prime}}^{N-N^{\prime}}\left(a_{H}^{2} q_{\perp}^{2}\right) \\
& \times \exp \left[-\frac{a_{H}^{2}}{4} q_{\perp}^{2}+i a_{H}^{2} q_{y}\left(k_{x}+\frac{q_{x}}{2}\right)\right]
\end{aligned}
$$

for $N^{\prime} \leqslant N$, where $q_{\perp}=\sqrt{q_{x}^{2}+q_{y}^{2}}$ and $L_{N^{\prime}}^{N-N^{\prime}}(x)$ is a Laguerre polynomial.

Now considering the case of a high magnetic field and low temperature, $\omega_{H} \gg T$, all the bosons are in the lowest Landau level, i.e., $N=N^{\prime}=0$. Also at the condensation point, as has been previously noted, the chemical potential $\mu$ is at the edge of the spectrum, $E_{c}$, which for free bosons is $E_{c}=\omega_{H} / 2$. Taking the free particle Matsubara Green's functions, as a first-order approximation for the polarization loop, we obtain

$$
\begin{aligned}
\Pi\left(\mathbf{q}, i \Omega_{n}\right)= & \sum_{k_{z}, k_{x}} \frac{n\left[\left(k_{z}+q_{z}\right)^{2} / 2 m\right]-n\left(k_{z}^{2} / 2 m\right)}{i \Omega_{n}-k_{z} q_{z} / m-k_{z}^{2} / 2 m} \\
& \times \exp \left(-a_{H}^{2} q_{\perp}^{2} / 2\right),
\end{aligned}
$$

where the Bose distribution function is

$$
n(\epsilon)=\frac{1}{e^{\epsilon / T}-1}
$$

We note that the sum rule, Eq. (19), for free particles, after summation over Matsubara frequencies is

$$
\sum_{k_{z}, k_{x}} n\left(\frac{k_{z}^{2}}{2 m}\right)=n_{B} .
$$

For ultralow temperatures we can approximate the Bose distribution function to a $\delta$ function of weight $n_{B}$ at zero momentum,

$$
n\left(\frac{k_{z}^{2}}{2 m}\right) \approx \delta_{k_{z}, 0} 2 \pi a_{H}^{2} n_{B}
$$

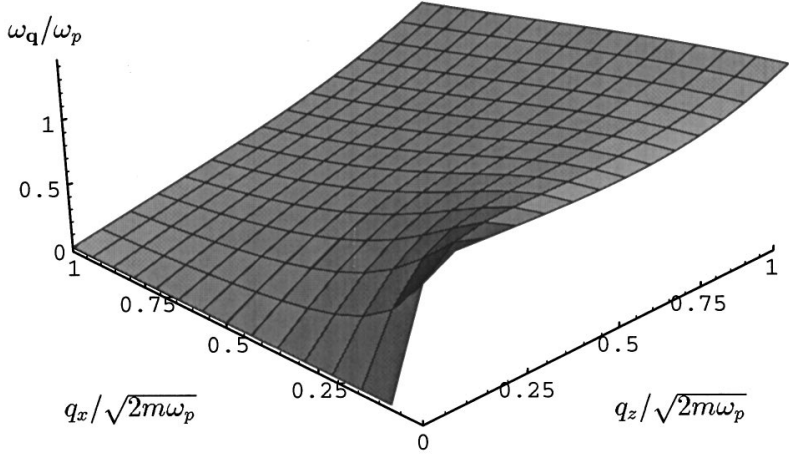

FIG. 3. Plasma frequency as a function of momentum.

The factor $2 \pi a_{H}^{2}$ arises from the summation over $k_{x}$,

$$
\sum_{k_{x}}=\frac{1}{2 \pi a_{H}^{2}} .
$$

This summation is finite because it is limited by the condition that the Landau eigenfunction lies within the normalized volume $(\equiv 1)$ being considered. This condition places a boundary on allowed values of $k_{x}$ as $y_{0}=k_{x} a_{H}^{2}$ is the center of the orbit.

Using the approximation in Eq. (45), the single-particle polarization loop

$$
\Pi\left(\mathbf{q}, i \Omega_{n}\right)=\frac{n_{B}}{m} \frac{q_{z}^{2} \exp \left(-a_{H}^{2} q_{\perp}^{2} / 2\right)}{\left(i \Omega_{n}\right)^{2}-q_{z}^{4} / 4 m^{2}}
$$

takes on the same form as for zero temperature. ${ }^{15}$ This gives the plasmon propagator as

$$
\begin{aligned}
\mathcal{D}\left(\mathbf{q}, i \Omega_{n}\right) & \\
= & \frac{4 \pi e^{* 2}}{q^{2}} \\
& \times \frac{\left(i \Omega_{n}\right)^{2}-q_{z}^{4} / 4 m^{2}}{\left(i \Omega_{n}\right)^{2}-q_{z}^{4} / 4 m^{2}-4 \pi e^{2} n_{B} q_{z}^{2} \exp \left(-a_{H}^{2} q_{\perp}^{2} / 2\right) / m q^{2}},
\end{aligned}
$$

where $q^{2}=q_{z}^{2}+q_{\perp}^{2}$.

The frequency of plasma excitations is given by the poles of the retarded plasmon propagator, which is found from the analytical continuation of the Matsubara plasmon propagator to the real axis,

$$
D^{R}(\mathbf{q}, \Omega)=\mathcal{D}\left(\mathbf{q}, i \Omega_{n} \rightarrow \Omega+i \delta\right) .
$$

The poles of the retarded plasmon propagator, and thus the plasma excitations, occur at

$$
\Omega \equiv \omega_{\mathbf{q}}=\left[\left(\frac{q_{z}^{2}}{2 m}\right)^{2}+\omega_{p}^{2} \frac{q_{z}^{2}}{q^{2}} \exp \left(-\frac{1}{2} a_{H}^{2} q_{\perp}^{2}\right)\right]^{1 / 2} .
$$

For $q_{\perp}=0$ this is the same as the nonmagnetic case, $\Omega \sim \omega_{p}$ for $q_{z} \rightarrow 0$. However, for $q_{\perp} \neq 0$ the plasmon is now gapless and sound like for low momenta, (Fig. 3). The effect of the magnetic field is to confine the bosons to their Landau orbitals along the $z$ axis. This allows freedom of movement along the $z$ axis, but restricts movement perpendicular to the $z$ axis, making the system stiffer to perturbations in that di- 


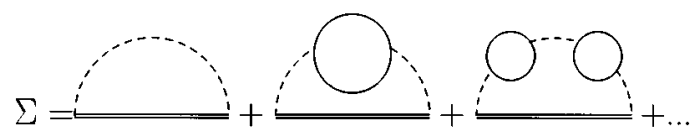

FIG. 4. Coulomb self-energy.

rection. So the polarization perpendicular to the $z$ axis is lowered in a magnetic field, resulting in soundlike, rather than plasmalike, excitations.

The approximation used in Eq. (45) produces a result similar to the zero-temperature superfluid Bogoliubov (3D) spectrum. ${ }^{3}$ The validity of this approximation arises from the ultrahigh magnetic field, which lowers the condensation temperature. This ensures that all of the bosons are at very low energies even in the normal state, hence validating Eq. (45).

\section{CONDENSATION OF CBG DUE TO THE COULOMB SCATTERING}

The self-energy due to boson-boson scattering in the Matsubara representation is

$$
\Sigma_{\nu}\left(i \Omega_{n}\right)=-T \sum_{\mathbf{q}, \nu^{\prime}, i \Omega_{m}}\left|I_{\nu}^{\nu^{\prime}}(\mathbf{q})\right|^{2} \mathcal{D}\left(\mathbf{q}, i \Omega_{m}\right) \mathcal{G}_{\nu^{\prime}}\left(i \Omega_{n}+i \Omega_{m}\right)
$$

This expression includes all "bubble" diagrams which is a fair approximation for the long-range Coulomb potential (Fig. 4).

As the density of states, Eq. (20), is obtained from the retarded Green's functions, we will transform this Matsubara representation using

$$
\mathcal{G}_{\nu}\left(i \Omega_{n}\right)=\frac{1}{2 \pi} \int d \epsilon^{\prime} \frac{A_{\nu}\left(\epsilon^{\prime}\right)}{i \Omega_{n}-\epsilon^{\prime}},
$$

where $A_{\nu}(\epsilon)$ is the spectral function defined as

$$
A_{\nu}(\epsilon)=\left[G_{\nu}^{A}-G_{\nu}^{R}\right]=-2 \operatorname{Im} G_{\nu}^{R} .
$$

Note that $G_{\nu}^{A}=G_{\nu}^{R^{*}}$.

Summing over the Matsubara frequencies the resulting equation is

$$
\begin{aligned}
\Sigma_{\nu}\left(i \Omega_{n}\right)= & \sum_{\mathbf{q}, \nu^{\prime}}\left|I_{\nu}^{\nu^{\prime}}(\mathbf{q})\right|^{2} \frac{1}{2 \pi} \int d \epsilon^{\prime} \frac{4 \pi e^{* 2}}{q^{2}} A_{\nu^{\prime}}\left(\epsilon^{\prime}\right) \\
& \times\left[\frac { \omega _ { p } ^ { 2 } q _ { z } ^ { 2 } \operatorname { e x p } ( - a _ { H } ^ { 2 } q _ { \perp } ^ { 2 } / 2 ) } { 2 q ^ { 2 } \omega _ { \mathbf { q } } } \left(\frac{n\left(\omega_{\mathbf{q}}\right)}{i \Omega_{n}-\epsilon^{\prime}+\omega_{\mathbf{q}}}\right.\right. \\
& \left.+\frac{n\left(\omega_{\mathbf{q}}\right)+1}{i \Omega_{n}-\epsilon^{\prime}-\omega_{\mathbf{q}}}\right) \\
& \left.+\frac{\left[\left(\epsilon^{\prime}-i \Omega_{n}\right)^{2}-q_{z}^{4} / 4 m^{2}\right] n\left(\epsilon^{\prime}\right)}{\left(\epsilon^{\prime}-i \Omega_{n}-\omega_{\mathbf{q}}\right)\left(\epsilon^{\prime}-i \Omega_{n}+\omega_{\mathbf{q}}\right)}\right],
\end{aligned}
$$

where $\omega_{q}$ is the plasma frequency for momentum $\mathbf{q}=\left\{q_{z}, q_{\perp}\right\}$ given by Eq. (50). This can be simplified for the case of ultralow temperatures when the only remaining significant term is from the spontaneous emission of plasmons.
All terms containing the Bose-distribution factor $n(\epsilon)$ are removed, leaving the zero-temperature result as this is the most significant part.

The retarded self-energy is easily derived from the Matsubara self-energy,

$$
\Sigma_{\nu}^{R}(\epsilon)=\Sigma_{\nu}(i \Omega \rightarrow \epsilon+i \delta) .
$$

The result is similar to the case of scattering from the impurities if we take the plasma frequency in the denominator, $\epsilon-\epsilon^{\prime}-\omega_{\mathbf{q}}+i \delta$, as zero. This is an elastic scattering approximation, which is reasonable if the characteristic plasma frequency is small compared with the characteristic broadening of the Landau level. We will show this to be true for the condition $T_{c_{0}} \ll \omega_{H}$.

The spectral function in terms of the retarded self-energy is

$$
A_{\nu}(\epsilon)=-2 \operatorname{Im} \frac{1}{\epsilon-k_{z}^{2} / 2 m-\Sigma_{\nu}^{R}(\epsilon)+\mu} .
$$

We now have a self-consistent equation for the selfenergy,

$$
\sum_{k_{z}}^{R}(\epsilon)=e^{* 2} \omega_{p}^{2} \sum_{\mathbf{q}} \int d \epsilon^{\prime} \frac{q_{z}^{2} \exp \left(-a_{H}^{2} q_{\perp}^{2}\right)}{\left(q_{z}^{2}+q_{\perp}^{2}\right)^{2} \omega_{\mathbf{q}}} \frac{A_{k_{z}+q_{z}}\left(\epsilon^{\prime}\right)}{\left(\epsilon-\epsilon^{\prime}+i \delta\right)} .
$$

Extending the summation over $\mathbf{q}$ to an integral,

$$
\sum_{\mathbf{q}}=\frac{1}{(2 \pi)^{2}} \int d q_{z} \int d q_{\perp} q_{\perp},
$$

we can begin to build an approximate solution. For high magnetic fields $a_{H}$ is small and for low $q_{z}$ the integral $d q_{\perp}$ can be approximated as

$$
\int d q_{\perp} \frac{q_{\perp} q_{z}^{2} e^{-a_{H}^{2} q_{\perp}^{2}}}{\left(q_{z}^{2}+q_{\perp}^{2}\right)^{2} \omega_{\mathbf{q}}} \approx \frac{1}{\omega_{p}} .
$$

To eliminate the chemical potential from the equation we choose that $\epsilon$ is zero at the mobility edge, i.e., $E_{c}=0$, and thus $\mu$ is also zero at the condensation temperature. As in the case of impurity scattering of bosons we expect the selfenergy to be momentum independent. The integration of $A_{k_{z}+q_{z}}\left(\epsilon^{\prime}\right)$ over $d q_{z}$ gives the density of states, which for a momentum-independent self-energy is

$$
\int d q_{z} A_{k_{z}+q_{z}}\left(\epsilon^{\prime}\right)=\sqrt{2 m} \operatorname{Im} \frac{4 \pi i}{\left[\epsilon^{\prime}-\Sigma^{R}\left(\epsilon^{\prime}\right)\right]^{1 / 2}} .
$$

Then the imaginary part of $\Sigma$ is obtained by integrating over the energy in Eq. (57) as

$$
\operatorname{Im} \Sigma^{R}(\epsilon)=-\frac{\sqrt{2}}{3^{1 / 4}} r_{s}^{3 / 4} \omega_{p}^{3 / 2} \operatorname{Im} \frac{i}{\left[\epsilon-\Sigma^{R}(\epsilon)\right]^{1 / 2}} .
$$

Despite the appearance of the minus sign in Eq. (61) the imaginary self-energy is positive. This is not obvious from Eq. (61), but comes from the relationship of $\operatorname{Im} \Sigma^{R}$ to the density of states, to be given later. As an exercise we can use 


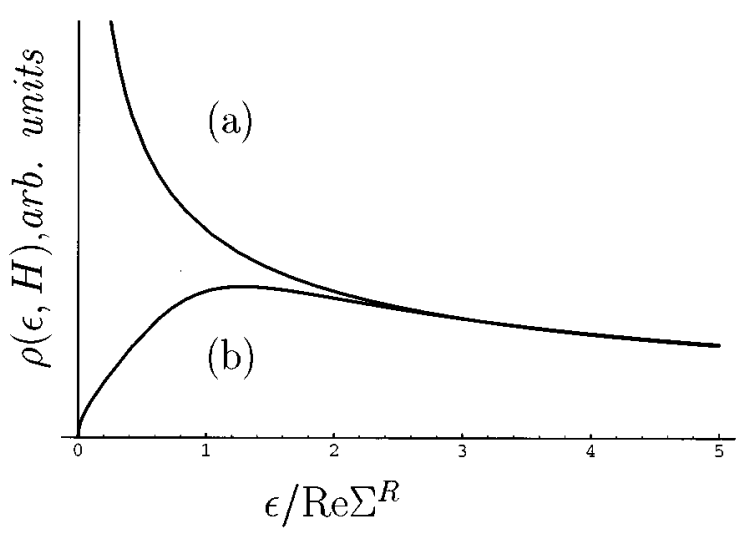

FIG. 5. Density of states in a magnetic field without (a) and with (b) the Coulomb interaction.

a first-order approximation for the self-energy, $\Sigma^{R}=+i \delta$. Substituting this into Eq. (61),

$$
\operatorname{Im} \frac{i}{\left[\epsilon-\Sigma^{R}(\epsilon)\right]^{1 / 2}}=-\frac{1}{\sqrt{\epsilon}},
$$

where the minus sign arises from taking the square root cut line on the positive real axis.

Expanding the cubic equation (61), and taking the limit of small $\operatorname{Im} \Sigma^{R}$ and small $\epsilon$, we find that for Eq. (61) to be satisfied in the lowest order, $\operatorname{Im} \Sigma^{R}=\epsilon=0$,

$$
\operatorname{Re} \Sigma^{R}(\epsilon)=\frac{\omega_{p} r_{s}^{1 / 2}}{(2 \sqrt{3})^{1 / 3}} .
$$

The next order in $\operatorname{Im} \Sigma^{R}$ and $\epsilon$ gives

$$
\operatorname{Im} \Sigma^{R}(\epsilon)=\left[\frac{12}{5(2 \sqrt{3})^{1 / 3}}\right]^{1 / 2} \omega_{p}^{1 / 2} r_{s}^{1 / 4} \sqrt{\epsilon} .
$$

The real part of $\Sigma^{R}$ can also be derived from the principal part of Eq. (57), which for low $\epsilon$ gives a constant of the same order as Eq. (63).

The density of states is directly related to the imaginary part of the self-energy,

$$
\rho(\epsilon, H)=\frac{1}{\pi^{2}} n_{B} \frac{\omega_{H}}{\omega_{p}} \operatorname{Im} \Sigma^{R}(\epsilon) .
$$

For high energies the density of states decreases as $1 / \sqrt{\epsilon}$, the same as the undamped result, Eq. (21). Using this to plot the density of states (Fig. 5), and remembering the sum rule

$$
n_{B}=-\frac{1}{(2 \pi)^{3} a_{H}^{2}} \int d q_{z} \int d \epsilon A_{\nu}(\epsilon) n(\epsilon),
$$

we can see that the low-energy solution, Eq. (64), is applied for the temperature range $T \ll \operatorname{Re} \Sigma^{R}$. The value of $H_{c 2}$ for which the sum rule can no longer be satisfied is then

$$
H_{c 2}=\frac{\Phi_{0}}{2 \pi \xi^{2}}\left(T_{c_{0}} / T\right)^{3 / 2}
$$

where now

$$
\xi \approx 0.92 n_{B}^{-1 / 3} r_{s}^{-1 / 2} \sqrt{\frac{e^{*}}{2 e}}
$$

is the coherence length due to the Coulomb scattering.

As was stated earlier, the elastic scattering approximation is only valid if the plasma frequency associated with the scattering is less than the width of the Landau level broadening, i.e., $\operatorname{Re} \Sigma^{R}$. This gives the condition

$$
\omega_{\mathbf{q}} \ll \operatorname{Re} \Sigma^{R} \approx \omega_{p} r_{s}^{1 / 2} .
$$

We can estimate the plasma frequency, from Eq. (50), as

$$
\omega_{\mathbf{q}} \approx \frac{q_{z}}{q_{x}} \omega_{p},
$$

with the momenta associated with the scattering process as $q_{x} \approx a_{H}^{-1}$ and $q_{z} \approx \sqrt{m \omega_{p} r_{s}^{1 / 2}}$. The estimation for $q_{z}$ comes as the momentum associated with the broadening, i.e., $q_{z}^{2} / 2 m \approx \operatorname{Re} \Sigma^{R}$. Thus the condition for using the elastic scattering approximation is

$$
\omega_{H} \gg \omega_{p} r_{s}^{-1 / 2} \approx T_{c_{0}} .
$$

In short the effect of including the interaction is to restore the density of states to its 3D form, and it is the energy dependence of the DOS which determines the temperature dependence of $H_{c 2}$. The broadening of the Landau levels is proportional to the interaction strength, i.e., $r_{s}$. The lower the interaction, the less the damping of the Landau levels and thus the lower $H_{c 2}$ is.

\section{CONCLUSION}

In contrast to the Fermi liquid, in which the long-range Coulomb interaction is screened and high-energy plasmons are irrelevant for low-frequency kinetics, allowance for the Coulomb interaction at finite temperatures in the $\mathrm{CBG}$ is a more complicated matter because plasmons and one-particle excitations are essentially the same in the long-wave limit. In this paper we derived the Bogoliubov-de Gennes-type equations for the $\mathrm{CBG}$ at finite temperatures and studied the Bose-Einstein condensation in an ultrahigh magnetic field. In contrast with the canonical GLAG theory the upper critical field of $\mathrm{CBG}$ is determined by the sum rule beyond the mean-field approximation. The damping of the Landau levels due to the scattering is a key feature allowing Bose-Einstein condensation in a magnetic field. We have extended the earlier results ${ }^{13,14}$ on the impurity scattering in CBG to very low temperatures, when the critical magnetic field is very high, so that the multiple impurity scattering becomes important. As a result the crossover from the $T^{-4.5}$ to the $T^{-0.5}$ exponent is found in the divergent $H_{c 2}(T)$ when the temperature is lowered. The plasmon dispersion as well as the BoseEinstein condensation due to Coulomb scattering is studied in an ultrahigh magnetic field as well. The plasmon is gapless. The Coulomb scattering results in the $T^{-1.5}$ exponent of $H_{c 2}$ at low temperatures. In general, the CBG has an unusual phase transition from the normal to the condensed phase in the magnetic field with remarkable positive curvature. At low temperatures we find $H_{c 2} \sim T^{-\nu}$. Depending on the tem- 
perature interval and impurity concentration the critical exponent $\nu$ varies from 0.5 up to 4.5 . We believe that the $\mathrm{CBG}$ is a relevant model of the ground state of high- $T_{c}$ cuprates with a very small coherence volume. If it is so, our predictions can be verified by the resistive and magnetic measurements in high magnetic fields.

\section{ACKNOWLEDGMENTS}

We acknowledge helpful discussions with our colleagues at IRC, Cambridge University, and at the Department of Physics, Loughborough University, and the Royal Society financial support for one of us (V.V.K.).
${ }^{1}$ A.S. Alexandrov and N.F. Mott, High Temperature Superconductors and Other Superfluids (Taylor \& Francis, London, 1994); Rep. Prog. Phys. 57, 1197 (1994).

${ }^{2}$ M.R. Schafroth, Phys. Rev. 100, 463 (1955).

${ }^{3}$ L.L. Foldy, Phys. Rev. 124, 649 (1961).

${ }^{4}$ N. Bogoliubov, J. Phys. (Moscow) 11, 23 (1947).

${ }^{5}$ R.F. Bishop, J. Low Temp. Phys. 15, 601 (1974).

${ }^{6}$ A.L. Fetter, Ann. Phys. (N.Y.) 64, 1 (1971).

${ }^{7}$ S.R. Hore and N.E. Frankel, Phys. Rev. B 12, 2619 (1975); 14, 1952 (1976).

${ }^{8}$ D.F. Hines and N.E. Frankel, Phys. Rev. B 20, 972 (1979).

${ }^{9}$ A. Gold, Z. Phys. B 83, 429 (1991).

${ }^{10}$ D.K. Lee and E. Feenberg, Phys. Rev. 137, A731 (1965).

${ }^{11}$ K.A. Brueckner, Phys. Rev. 156, 204 (1967).

${ }^{12}$ C-W. Woo and S. Ma, Phys. Rev. 159, 176 (1967).

${ }^{13}$ A.S. Alexandrov, Ph.D. thesis, MIFI, Moscow, 1984; Phys. Rev. B 48, 10571 (1993).

${ }^{14}$ A.S. Alexandrov et al., Phys. Rev. Lett. 76, 983 (1996) and references therein.

${ }^{15}$ A.S. Alexandrov and W.H. Beere, Phys. Rev. B 51, 5887 (1995).

${ }^{16}$ A.S. Alexandrov, D.A. Samarchenko, and S.V. Traven, Zh. Eksp.
Teor. Fiz 93, 1007 (1987) [Sov. Phys. JETP 66, 567 (1987)].

${ }^{17}$ H. Ehrenreich and L.M. Scwartz, in Solid State Physics, edited by H. Ehrenreich, F. Zeitz, and D. Turnbull (Academic, New York, 1976), Vol. 31, p. 149.

${ }^{18}$ S.F. Edwards, Philos. Mag. 31020 (1958).

${ }^{19}$ A.A. Abrikosov, L.P. Gorkov, and I.E. Dzyaloshinskii, Quantum Field Theoretical Methods in Statistical Physics (Pergamon, New York, 1965).

${ }^{20}$ A.A. Abrikosov, Fundamentals of the Theory of Metals (Elsevier, New York, 1988), pp. 381-389.

${ }^{21}$ Yu.A. Bychkov, Zh. Eksp. Teor. Fiz. 39, 1401 (1960) [Sov. Phys. JETP 12, 977 (1961)].

${ }^{22}$ V.G. Skobov, Zh. Eksp. Teor. Fiz. 40, 1446 (1961) [Sov. Phys. JETP 13, 1014 (1961)]; 37, 1467 (1959) [10, 1039 (1959)]; 38, 1304 (1960) [11, 941 (1960)].

${ }^{23}$ L.I. Magarill and S.K. Savvinykh, Zh. Eksp. Teor. Fiz. 60, 175 (1971) [Sov. Phys. JETP 33, 97 (1971)]; Fiz. Tverd. Tela 13, 1858 (1971) [Sov. Phys. Solid State 13, 1558 (1972)].

${ }^{24}$ R. Kubo, S.J. Miyake, and N. Hashitsume, in Solid State Physics, edited by H. Ehrenreich, F. Zeitz, and D. Turnbull (Academic, New York, 1965), Vol. 17, p. 317. 